\title{
Single-Site Laparoscopic Colectomy in a Patient With Situs Inversus Totalis
}

\author{
Yasuo Sumi, MD, PhD, Takeru Matsuda, MD, PhD, Kimihiro Yamashita, MD, PhD, \\ Hiroshi Hasegawa, MD, PhD, Taro Oshikiri, MD, PhD, Tetsu Nakamura, MD, PhD, \\ Satoshi Suzuki, MD, PhD, Yoshihiro Kakeji, MD \\ Division of Minimally Invasive Surgery, Department of Surgery, Kobe University Graduate School of Medicine, Kobe, \\ Japan (Dr Sumi).
}

Division of Gastrointestinal Surgery, Department of Surgery, Kobe University Graduate School of Medicine, Kobe, Japan (Matsuda, Yamashita, Hasagawa, Oshikiri, Nakamura, Suzuki, and Kakeji).

\begin{abstract}
Introduction: Situs inversus totalis (SIT) is a rare congenital anomaly in which the abdominal and thoracic cavity structures are located opposite to their usual positions. Occasionally, patients with SIT, who have malignant tumors, are encountered. Recently, several laparoscopic operations have been reported in patients with SIT.

Case Description: We report a case of a 74-year-old woman with SIT who developed cecum cancer. Single-site laparoscopic colectomy (SSLC) with radical lymphadenectomy was successfully performed with careful consideration of the mirror-image anatomy. The techniques themselves were not different from those used in ordinary cases.

Conclusion: Thus, SSLC for colon cancer in a patient with SIT can be performed safely by a skilled surgeon after thorough preoperative planning, including assessment of the anomaly. This procedure therefore remains a feasible option, even for patients with SIT, allowing them to benefit from minimally invasive surgery.
\end{abstract}

Key Words: Right colon cancer, Single-site laparoscopic surgery, Situs inversus totalis.

Citation Sumi Y, Matsuda T, Yamashita K, Hasegawa H, Oshikiri T, Nakamura T, Suzuki S, Kakeji Y. Single-site laparoscopic colectomy in a patient with situs inversus totalis. CRSLS e2017.00034. DOI: 10.4293/CRSLS.2017.00034.

Copyright $\odot 2017$ by SLS, Society of Laparoendoscopic Surgeons. This is an open-access article distributed under the terms of the Creative Commons Attribution-Noncommercial-ShareAlike 3.0 Unported license, which permits unrestricted noncommercial use, distribution, and reproduction in any medium, provided the original author and source are credited.

Disclosures: none reported.

Address correspondence to: Yasuo Sumi, MD, PhD, Division of Minimally Invasive Surgery, Department of Surgery, Kobe University Graduate School of Medicine,

7-5-2 Kusunoki-cho, Chuo-ku, Kobe 650-0017, Japan. Telephone: +81-78-382-5925, Fax: +81-78-382-5939, E-mail: sumiy@med.kobe-u.ac.jp

\section{INTRODUCTION}

Situs inversus totalis (SIT) is a rare congenital anomaly with complete right-left inversion of thoracic and abdominal viscera. Laparoscopic surgery for SIT is considered to be more difficult because of this mirror-image anatomy. Although the use of this surgery for colorectal cancer is rapidly becoming widespread, we found only 8 case reports in patients with SIT. ${ }^{1-8}$ To our knowledge, thus far, there is only 1 case report of single-site laparoscopic surgery in SIT. ${ }^{8}$ We present another case.

\section{CASE REPORT}

A 74-year-old woman with SIT visited the Department of Internal Medicine in our hospital, reporting bloody stool.
A colonoscopy revealed a type 2 tumor in the cecum, diagnosed as a well-differentiated adenocarcinoma by biopsy (Figure 1). The patient was admitted for further evaluation and surgical treatment. Laboratory examination confirmed anemia (red blood cell count, $348 \times 104 / \mathrm{mm}^{2}$; hemoglobin, $10.8 \mathrm{~g} / \mathrm{dL}$; and hematocrit, 33.7\%). Serum carcinoembryonic antigen was not elevated $(2.6 \mathrm{ng} / \mathrm{mL}$; normal range, $0-5 \mathrm{ng} / \mathrm{mL}$ ). Chest radiography showed dextrocardia and a right subphrenic gastric bubble (Figure 2A). Abdominal computed tomography revealed complete transposition of the abdominal viscera, confirming SIT (Figure 2B). Stereoscopic imaging of the vessels by 3-dimensional computed tomography (3D-CT) showed no abnormal vascularization (Figure 3). According to 


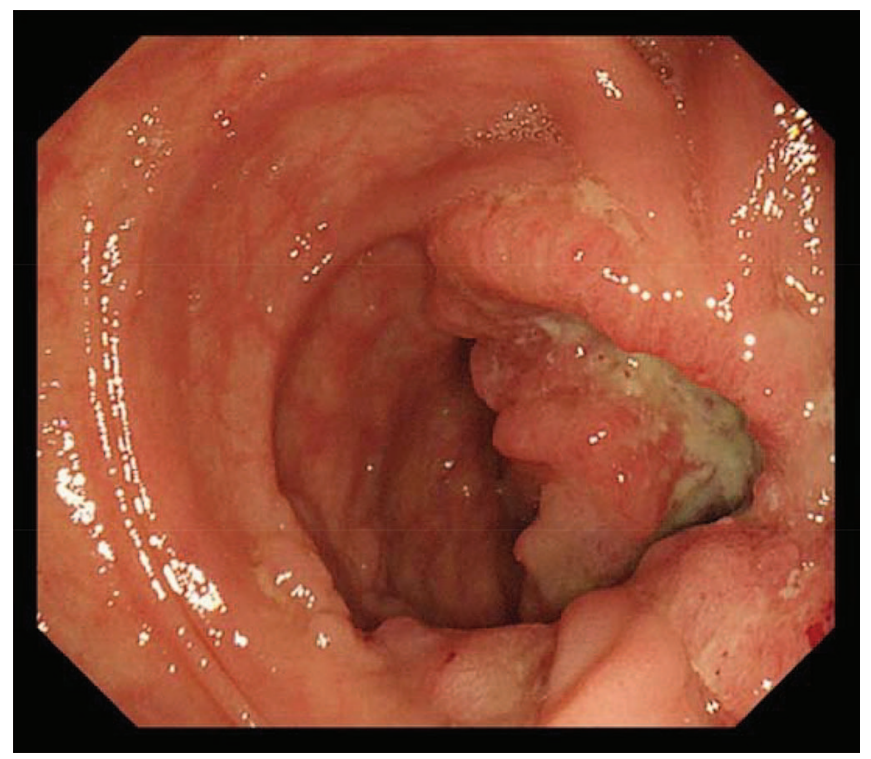

Figure 1. Colonoscopy showing an ulcerated lesion.

these findings, we diagnosed T3 N1 M0 Stage IIIa colon cancer and single-incision laparoscopic colectomy with radical lymphadenectomy was performed.

\section{Surgical Procedure}

With the patient under general anesthesia and supine, a Lap Protector Mini (Hakkou Shoji, Japan) was inserted through a $3.5-\mathrm{cm}$ transumbilical incision with the patient. An EZ-access device (Hakkou Shoji, Japan) was mounted onto the Lap protector, and two 5-mm ports and one 10-mm port were used (Figure 4). An advanced 10-mm flexible 3-D laparoscope with wide optic angle (Olympus, Tokyo, Japan) was used for visualization, which revealed that the cecum and ascending colon were situated at the left. Neither liver metastasis nor peritoneal dissemination was detected. The tumor was located at the cecum. The left-sided colon was mobilized with a medial approach. The ileocolic vessels were divided at their root, and all of the soft tissues anterior to the superior mesenteric vein were completely removed to perform D3 lymph node dissection (Figure 5, 6). After mobilization up to the ascending colon, dissection and anastomosis were performed extracorporeally with a functional end-to-end stapling method. Operating time was $153 \mathrm{~min}$ and blood loss was negligible. According to the Japanese classification of colorectal carcinoma, macroscopically, the tumor was a $60 \times 45-\mathrm{mm}$ type 2 lesion in the cecum. Histological examination of the resected specimen revealed a moderately differentiated adenocarcinoma (T4N2M0, Stage IIIb). After an uneventful postoperative course, the patient was started on a clear liquid diet on postoperative day 4, according to the clinical regimen at our hospital, and was discharged on postoperative day 8 . The patient received adjuvant chemotherapy for six months and did well without recurrence at 12 months.

\section{DISCUSSION}

The incidence of SIT is 1 in 5,000-20,000. Cardiovascular malformation (8\%) and bronchiectasis (10\%) are often present. With SIT, abnormal vascularization of the arteries and veins is common; therefore, preoperative confirmation of any abnormal vascularization is very important. Particularly with laparoscopic surgery, it is important to determine the presence of vascular anomalies on preoperative CT or angiography. ${ }^{9}$ We found only 8 cases of laparoscopic surgery for colorectal malignancy complicated by SIT reported in the English literature, and only one of them involved treatment with single-site laparoscopic surgery. ${ }^{1-8}$

Laparoscopic surgery is considered to be more difficult in patients with SIT than in other patients because of the mirror-image anatomy. It is therefore important for the safe and effective performance of laparoscopic surgery in patients with SIT to first undertake a preoperative examination, because the frequency of abnormal vascularization of arteries and veins is high, in addition to the problems posed by the mirror-image presentation of the viscera. In the case presented herein, stereoscopic imaging was undertaken with 3D-CT to provide a satisfactory overview of the vessels. We consider that the manner in which laparoscopic devices are handled in cases of SIT is not very different from the regular situation, because the operator does not notice the mirror-image positioning so much in laparoscopic surgery, which is performed in the very narrow area visible by laparoscope. We have noted this in the case reports of laparoscopic surgery for gastric and colon cancer in patients with SIT.6,10 Thus, provided the surgeon possesses fundamental laparoscopic surgical skills in terms of eye-hand coordination and bimanual coordination, special approaches are not necessary, even in a case of SIT.

However, as with any laparoscopic procedure, it is important to adhere to the fundamentals of careful handling of the device and to keep the operative field dry. In fact, lymph node dissection in our patient with SIT seemed unexpectedly easier because the direction of dissection, the energy device for a right-handed surgeon was parallel, and the mirror-image placement of the organs 

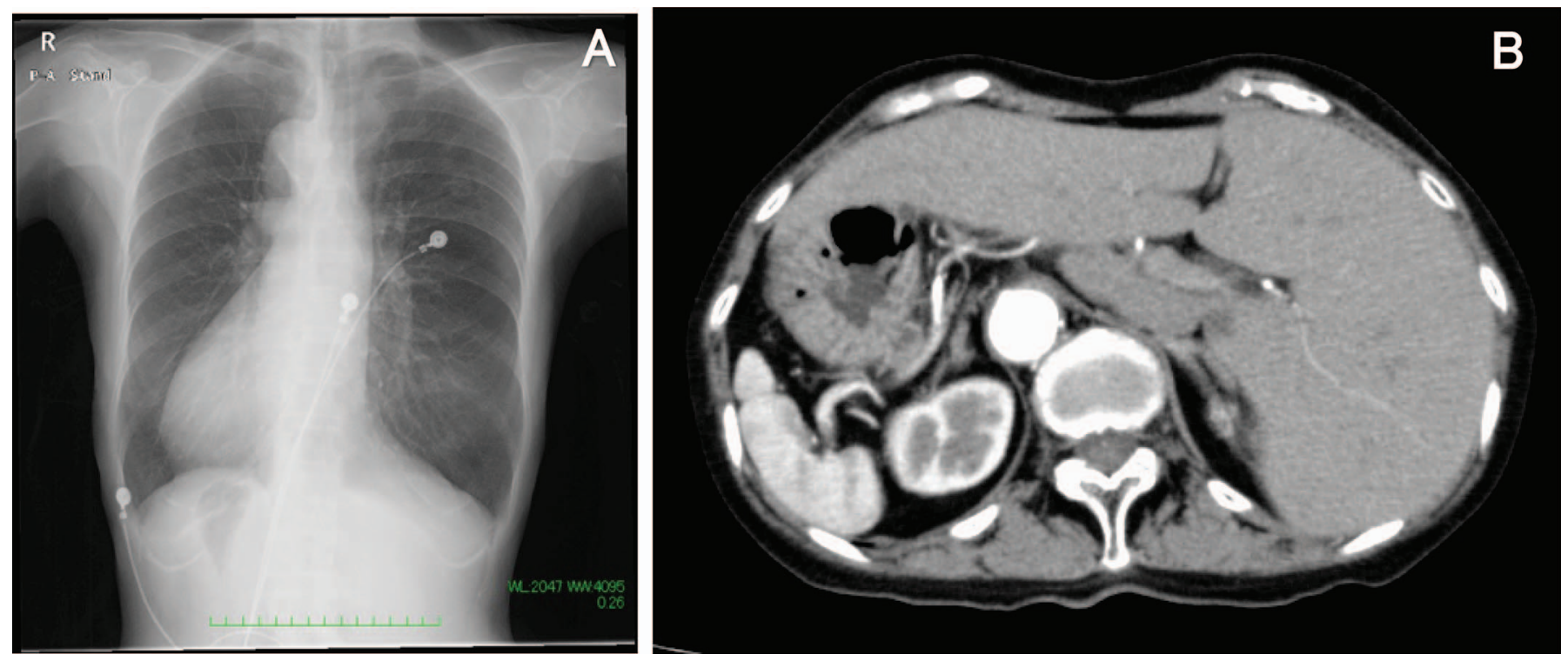

Figure 2. Chest radiography $\mathbf{A}$, and abdominal computed tomography $\mathbf{B}$. A, Dextrocardia and a right subphrenic gastric bubble are evident. B, Complete transposition of abdominal viscera is shown, confirming situs inversus totalis.

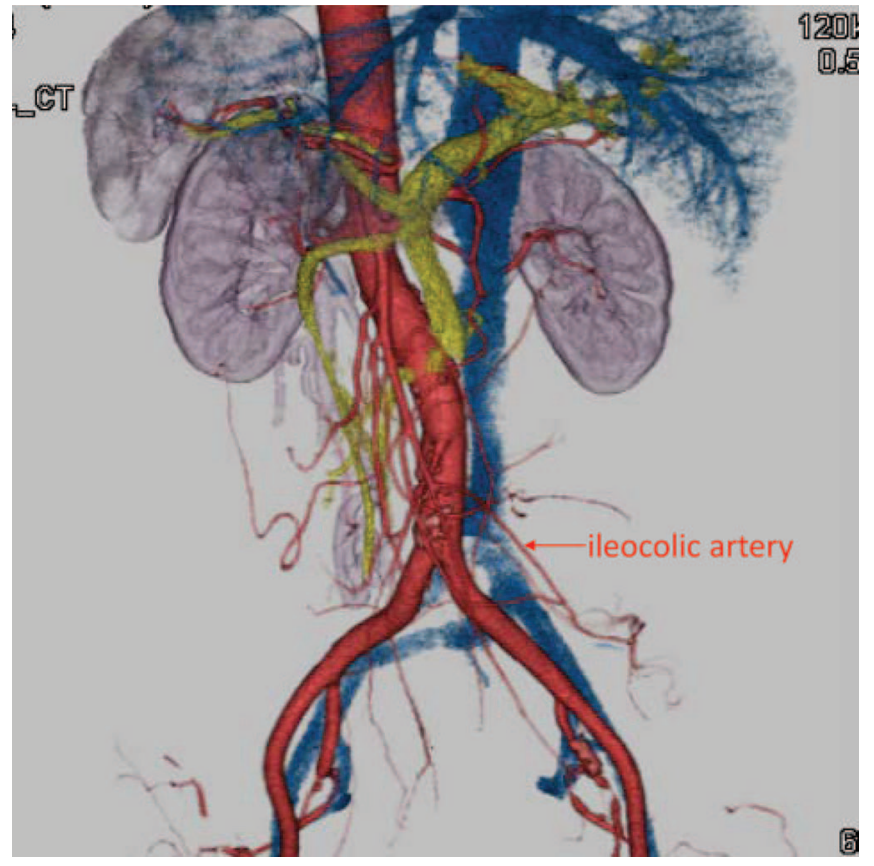

Figure 3. Three-dimensional CT. 3D-CT showed no abnormal vascularization except complete transposition of vessels.

made access for right-side colon cancer less problematic (Figure 7).

Nonetheless, SSLC is a challenging procedure because the movement of forceps is limited, and it is necessary to be well-practiced in their handling. We therefore believe that

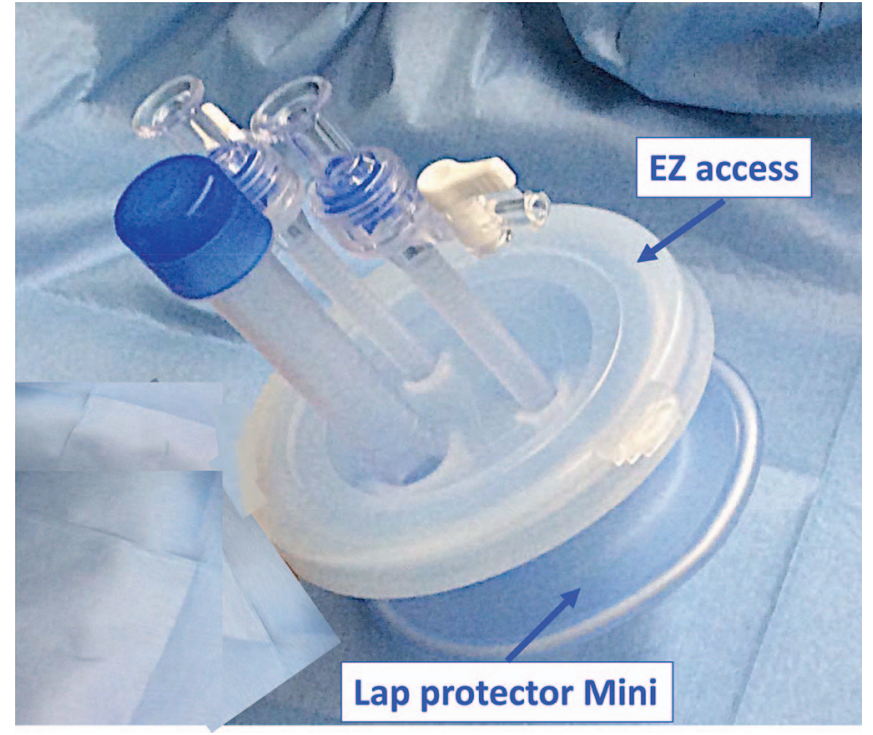

Figure 4. EZ access and lap protector mini.

SSLC should be performed by expert surgeons skilled in advanced techniques, such as those holding an Endoscopic Surgical Skill Qualification System certificate approved by the Japan Society for Endoscopic Surgery. ${ }^{11}$ We have already introduced SSLC for right colon cancer into our clinical practice since 2011 and have experience with enough cases where this is the surgical method of choice. As we now have reports of 2 cases of laparoscopic surgery for SIT, we can confirm that SSLC is also the best choice for these patients. 


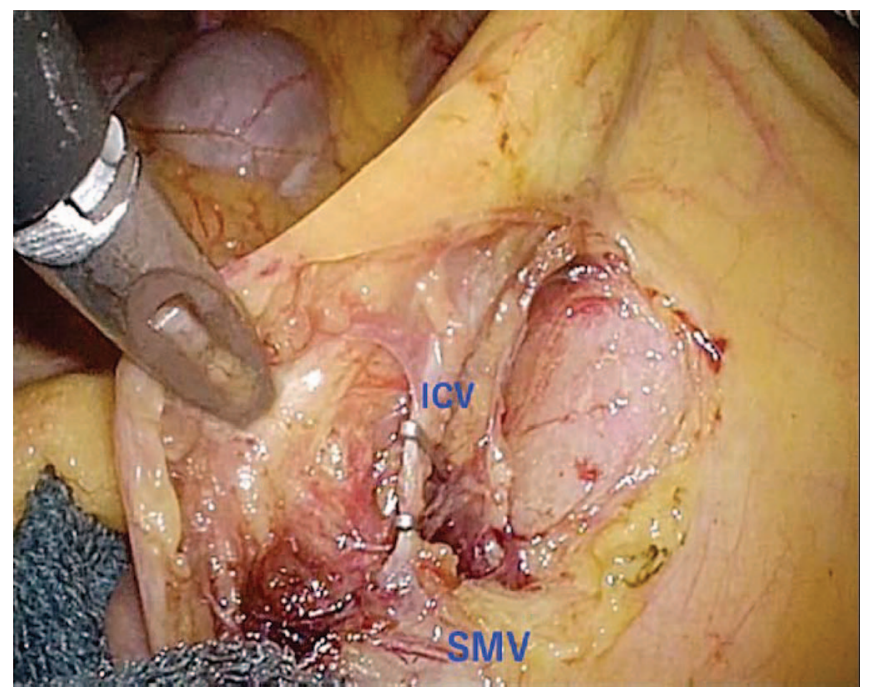

Figure 5. The root of the ileocecal vein.

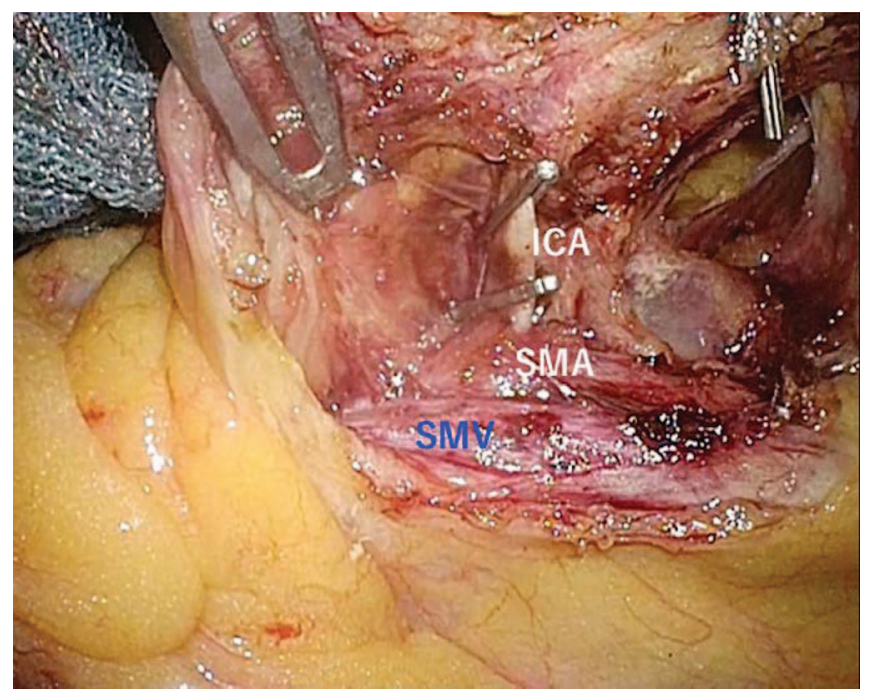

Figure 6. The root of the ileocecal artery.

In summary, SSLC for colon cancer in patients with SIT can be safely performed by a skilled surgeon after thorough preoperative planning, including assessment of the anomaly. This procedure is a feasible option for patients to benefit from minimally invasive surgery.

\section{References:}

1. Kim HJ, Choi GS, Park JS, et al. Laparoscopic right hemicolectomy with D3 lymph node dissection for a patient with situs inversus totalis: report of a case. Surg Today. 2011;41:15381542.

2. Choi SI, Park SJ, Kang BM, et al. Laparoscopic abdominoperineal resection for rectal cancer in a patient with situs

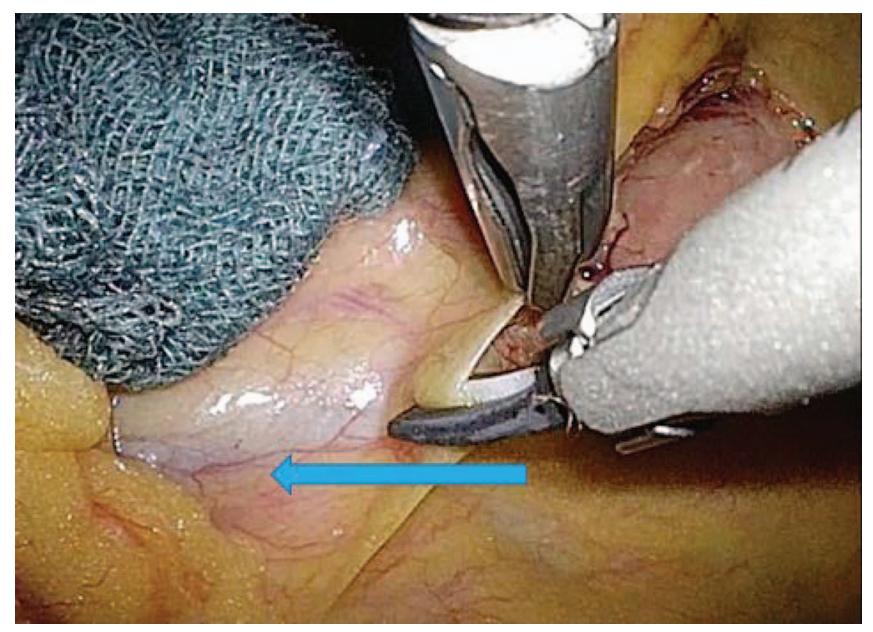

Figure 7. The direction of dissection and the energy device are in parallel.

inversus totalis. Surg Laparosc Endosc Percutan Tech. 2011; 21:e87-e90.

3. Huh JW, Kim HR, Cho SH, et al. Laparoscopic total mesorectal excision in a rectal cancer patient with situs inversus totalis. J Korean Med Sci 2010;25:790-793.

4. Fujiwara Y, Fukunaga Y, Higashino M, et al. Laparoscopic hemicolectomy in a patient with situs inversus totalis. World $J$ Gastroenterol 2007;13:5035-5037.

5. Kim YW, Ryu H, Kim DS, Kim IY. Double primary malignancies associated with colon cancer in patients with situs inversus totalis; two case reports. World J Surg Oncol. 2011;23109.

6. Sumi Y, Tomono A, Suzuki S, Kurda D, Kakeji Y. Laparoscopic hemicolectomy in a situs inversus totalis after open distal gastrectomy. World J Gastrointest Surg. 2013;5:22-26.

7. Yaegashi M, Kimura T, Sakamoto T, Sato T, Kawasaki Y, Otsuka K, Wakabayashi G. Laparoscopic sigmoidectomy for a patient with situs inversus totalis: effect of changing operator position. Int Surg. 2015;100:638-642.

8. Hirano Y, Hattori M, Douden K, Hashizume Y. Single-incision laparoscopic surgery for colon cancer in patient with situs inversus totalis: report of a case. Indian J Surg. 2015 77(Suppl 1):26-28.

9. Nursal TZ, Baykal A, Iret D, Aran O. Laparoscopic cholecystectomy in a patient with situs inversus totalis. J Laparoendosc Adv Surg Tech A. 2001;11:239-241.

10. Sumi Y, Maehara T, Matsuda Y, et al. Laparoscopy-assisted distal gastrectomy in a patient with situs inversus totalis. JSLS. 2014;18:314-318.

11. Mori T, Kimura T, Kitajima M. Skill accreditation system for laparoscopic gastroenterologic surgeons in Japan. Minim Invasive Ther Allied Technol. 2010;19:18-23. 